\title{
Quantitative Assessment of Two Skeletonization Algorithms Adapted to Rectangular Grids
}

\author{
M. CIUC, D. COQUIN, Ph. BOLON \\ Laboratoire d'Automatique et de Micro-Informatique Industrielle \\ LAMIU/CESALP - Université de Savoie, BP 806, F. 74016 Annecy Cedex (France) \\ e-mail: coquin @esia.univ-savoie.fr
}

\begin{abstract}
In this paper, an adaptation to rectangular grids of two skeletonization algorithms is presented. Skeletonizations are quantitatively compared by using Baddeley's distance between the original pattern and the one reconstructed from the skeleton.
\end{abstract}

Keywords: skeletonization, rectangular grids, Baddeley distance

\section{Introduction}

Among the different approaches to skeletonize objects, there are the methods based on a distance transformation. A Distance Map (DM) of the object is computed before extracting the skeleton, each pixel in the object being assigned to a label which represents the nearest distance of the pixel to the complement of the object (i.e. background). This property is useful, since it allows reconstruction of the pattern starting from its skeleton.

Several skeletonization algorithms based on a distance transformation have been developed. Most of them treat the case of images digitized on a square grid. However, most industrial vision systems digitize images with sampling steps which are different, depending on whether they are in rows or columns. Hence, images are digitized on a rectangular grid. To avoid the resampling of such images, algorithms which compute the distance map of a binary object from the background with respect to this particular shape of the pixel have been introduced. Skeletonization methods proposed for a square grid need some modifications.

In this paper, we propose an adaptation of the cases of images digitized on a rectangular grid, to two methods. The first one, proposed by Arcelli and Sanniti di Baja in [2] is based on the extraction of ridge points. The second, proposed by Thiel in [1] is based on the extraction of the centres of maximal discs by means of look-up table.

Results and comparisons between the two methods are provided in section 6 .

\section{Distance map on rectangular grids}

Many methods to extract the distance map of a binary object in an image have been developed: the city-block distance $\left(d_{4}\right)$ [9], the chessboard distance $\left(d_{8}\right)$ [3], the chamfer distance [1]. They are characterized by a local mask and are computed within a two raster-scan of the original binary image, regardless of the size of the object. The best approximation of the Euclidean distance is obtained by chamfer distances. This makes the result less dependent on rotation and scaling of the object than the other ones. Moreover, chamfer distances can be adapted to rectangular grids. The coefficients a,b,c,d,e (fig. 1) are optimized according to the method proposed in [4]. 


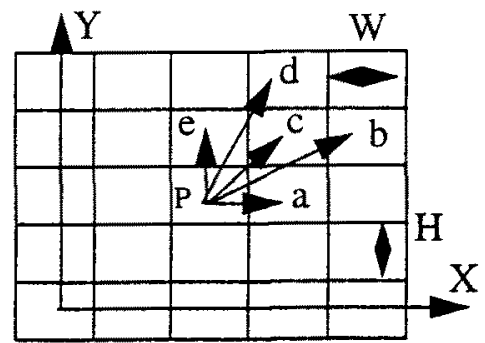

Fig. 1. real coefficients on rectangular grid, $\mathbf{W}=$ pixel width, $\mathbf{H}=$ pixel height

\begin{tabular}{|c|c|c|c|c|}
\hline & D & & $\bar{D}$ & \\
\hline \multirow[t]{2}{*}{$\bar{B}$} & $\overline{\mathrm{C}}$ & $\bar{E}$ & $\bar{C}$ & $\bar{B}$ \\
\hline & $\mathrm{A}$ & $\mathbf{p}$ & $\mathrm{A}$ & \\
\hline \multirow[t]{2}{*}{$\bar{B}$} & $\bar{C}$ & $\overline{\mathrm{E}}$ & $\overline{\mathrm{C}}$ & $\bar{B}$ \\
\hline & $\bar{D}$ & & $\bar{D}$ & \\
\hline
\end{tabular}

Fig. 2. $5 \times 5$ chamfer mask used for computation of the DM on rectangular grid

Subsequently, we use a $5 \times 5$ chamfer mask for the computation of the Distance Map. DM can be obtained with either a sequential or a parallel algorithm [5].

For reasons of computation time and memory availability, integer coefficients $A, B, C, D, E$, obtained by means of scaling and rounding the real ones are used instead.

$$
\{A, B, C, D, E\}=\operatorname{round}(\{a, b, c, d, e\} . N)
$$

where $N$ is chosen with regard to the errors that appear by rounding and to the limits of memory required to store the distance map.

\section{Centre of Maximal Discs extraction}

In this section, the method of Thiel [1] is extended to the case of a rectangular grid. Notations and definitions are kept. We use the same notation for pixels as well as for their labels. Any pixel $\mathrm{p}$ belonging to the object can be regarded as a centre of a disc $D_{R p}$ which includes each pixel q such that: $\quad \mathbf{d c}(\mathbf{p}, \mathbf{q})<\mathbf{R p}$ $\operatorname{dc}(p, q)$ denotes the chamfer distance between pixels $p$ and $q$.

Pixels in the DM for which the associated disc is not completely covered by any other disc is declared Centre of a Maximal Disc (CMD) and is marked as belonging to the Median Axis (MA). The set of CMD constructed in this way is sufficient to allow a perfect reconstruction of the original pattern. Hence, it is not minimal, since it contains pixels from the associated disc included not in another disc, but in the union of other discs. Our main goal is to extract the skeleton. Hence, these pixels must be kept as skeletal in order to achieve connectivity.

\subsection{Look-Up Table computation}

It was shown that, generally, it is sufficient to verify for each pixel the following condition:

$\mathrm{p} \in \mathrm{MA} \Leftrightarrow \forall \mathrm{i} \quad \mathrm{n}_{\mathrm{i}}(\mathrm{p}) \leq \mathrm{LUT}_{\mathrm{i}}(\mathrm{p}) \quad$ with $\mathrm{n}_{\mathrm{i}}(\mathrm{p})$ is the mask,

ed to the chamfer distance dc.

$\mathrm{LUT}_{\mathrm{i}}(\mathrm{p})$ is the look-up table associat-

For a very fast and easy computation, a look-up table is precalculated. For each possible label $p$, the LUT stores the minimal value $q$ that the pixel located in one of the five directions $A, B, C, D, E$ should have, such that the disc $D_{R q}$ completely overlaps $\mathrm{D}_{\mathrm{Rp}}$. 
For the computation of the LUT we used the method of Thiel adapted to the rectangular grid. The only difference due to this distinctive feature of the CDM is that the mask is no more 8-symmetrical, but 4-symmetrical. Instead of building up 1/8 of the distance image to the origin (gray part), we are obliged to construct a quarter of it in order to obtain correct results.

\begin{tabular}{|c|c|c|c|c|}
\hline 0 & 42 & 84 & 126 & 168 \\
\hline 20 & 47 & 86 & 128 & 170 \\
\hline 40 & 57 & 94 & 133 & 172 \\
\hline 60 & 77 & 104 & 141 & 180 \\
\hline 80 & 97 & 114 & 151 & 188 \\
\hline
\end{tabular}

Fig. 3. quarter of the distance image to the origine with $\mathrm{H}=1, \mathrm{~W}=2$ $(A=42, B=86, C=47, D=57, E=20$ )
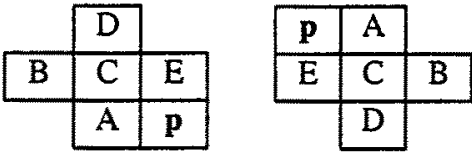

Fig. 4. Two quater of mask

The steps to be applied in order to compute the LUT (Fig. 5) are:

- step 1: We start from an image having all pixels set at a very high value, except for the origin, which is set at zero. The quarter of the image distance to the origin is computed by means of a single raster-scan, column by column from top-left to down-right with the quarter of the mask in Fig. 4 following the same principle as in the computation of the CDM (fig. 3) see section 3.2.

- step 2: The LUT is filled in a single raster-scan of the previous image too. For each pixel, labels of pixels located in each of the five directions as showed in Fig. 4 are inspected. These values, added with 1 , will be stored at the LUT entry corresponding to the label next to the current pixel's one.

- step 3: For each of the five directions $A, B, C, D, E$ the increasing order is established in the LUT.

\begin{tabular}{|c|c|c|c|c|c|}
\hline $\mathbf{p}$ & $\mathbf{a}$ & $\mathbf{b}$ & $\mathbf{c}$ & $\mathbf{d}$ & $\mathbf{e}$ \\
\hline $\mathbf{2 0}$ & $\mathbf{4 3}$ & 87 & 48 & 58 & 21 \\
\hline $\mathbf{4 0}$ & 48 & 95 & 58 & 78 & 41 \\
\hline $\mathbf{4 2}$ & 58 & 105 & 78 & 98 & 61 \\
\hline $\mathbf{4 7}$ & 85 & 129 & 87 & 98 & 61 \\
\hline $\mathbf{5 7}$ & 87 & 134 & 95 & 105 & 61 \\
\hline $\mathbf{6 0}$ & 95 & 142 & 105 & 115 & 78 \\
\hline $\mathbf{7 7}$ & 95 & 142 & 105 & 118 & 81 \\
\hline
\end{tabular}

Fig. 5. the beginning of LUT for $A=42, B=86, C=47, D=57, E=20,(H=1, W=2)$

\subsection{CMD extraction}

The set of CMD is extracted within a single raster-scan of the image containing the CDM by means of a single inspection of the neighbourhood of the pixels. For each pixel we inspect the labels of pixels located in one of the five directions $A, B, C, D, E$. If none of these pixels has a label which is equal to or greater than the LUT entry corresponding to the current pixel label in the same direction, then the current pixel is marked as a CMD (Fig. 6). The set of CMD is not sufficient to permit connectivity. Also saddle points, i.e. pixels which connect two parts of the DM with greater labels must be detected. The conditions a pixel must satisfy to be declared a saddle point are 
kept with no modifications from [1]. The set of CMD and saddle points represents the median axis (MA) of the object. Starting from it, skeletons will be obtained after the steps in section 5 are applied.

$\begin{array}{cccccc}0 & 20 & 20 & 40 & 57 & 77 \\ 20 & 40 & 40 & 57 & 77 & 97 \\ 40 & 57 & 60 & 77 & 97 & 14 \\ 57 & 60 & 77 & 80 & 97 & 100 \\ 40 & 40 & 57 & 60 & 77 & 86 \\ 20 & 20 & 40 & 40 & 57 & 60\end{array}$

Fig. 6. portion of the CDM with $A=20, B=86, C=47, D=57, E=20$.

gray pixels are detected as CMD

All median axis pixels, when detected, are introduced in a separate list, from which they will be directly accessed in order to increase the speed of the algorithm.

\section{Ridge points extraction}

In this section we will extend the method proposed by Arcelli and Sanniti di Baja in [2], to the case of rectangular grids. Notations and definitions are kept. As in the previous section, we will denote by $\mathrm{p}$ both the pixel and its label.

If we consider the DM as a landscape in three dimensions, the label of the pixel indicating its height, skeletal pixels are those located on the ridges, i.e. pixels having two neighbours with smaller labels at least in one direction. This is the principle of the method. Ridge points are detected in a one-pass raster-scan of the DM by means of a simple inspection of their neighbourhood [2]. Operators were applied only for pixels labelled more than one, i.e. for pixels not belonging to the 8-connected border of the pattern. For border pixels, two other sets of operands were especially designed. These operators can no more be applied in the case of rectangular grid DM (Fig. 7), since in this case there are two types of border pixels: the ones which are labelled $\mathrm{A}$, the others labelled E (Fig. 2).

$\begin{array}{ccccc}57 & 40 & 0 & 40 & 57 \\ 40 & 20 & 20 & 20 & 40 \\ 20 & 0 & 40 & 0 & 20 \\ 0 & 0 & 42 & 0 & 0\end{array}$

Fig. 7. neither of the gray pixels is detected as a ridge point with the operators designed for square grids

We propose the following modifications: we apply the operators described in [2] to the border pixels too. In addition, for pixels labelled $E$, we check if crossing number $\left(\mathrm{X}_{4}(\mathrm{p})>1\right)$ from the detection of saddle points in section 3.2 is satisfied, and we mark them too in this case. As we did in the CMD extraction case, pixels detected as ridge points are introduced in a separate list.

\section{Skeleton extraction}

Starting from the two sets of points determined in section 3 and 4 , called intrinsic skeletal pixels in what follows, the same steps are applied in order to obtain the skeleton. 


\subsection{The connection step}

The set of intrinsic skeletal pixels generally does not preserve the pattern connectivity order. Hence a connection step must be applied. It consists of growing paths following the steepest gradient direction from any intrinsic skeletal pixel.

Let $p$ be the current pixel. The gradient: is computed for all neighbours $p_{i}$ of pixel $p$, with:
$k=\frac{1}{A} \quad$ if $p_{i}$ is an $A$-neighbour
$k=\frac{1}{D} \quad$ if $p_{i}$ is a $D$-neighbour
$k=\frac{1}{B}$ if $p_{i}$ is a $B$-neighbour
$k=\frac{1}{E} \quad$ if $p_{i}$ is an $E$-neighbour

$k=\frac{1}{C} \quad$ if $p_{i}$ is a $C$-neighbour

$$
\operatorname{grad}_{p}\left(p_{i}\right)=k \cdot\left(p_{i}-p\right)
$$

The pixel $p_{i}$ which maximizes the gradient is marked as a skeletal pixel too (it is called an induced skeletal pixel) and we continue to grow the path starting from it. Path growing stops when either the pixel which maximizes the gradient is already marked, or all the gradients computed are negative, i.e. a local maximum in the CDM is reached.

If a B-neighbour or a D-neighbour is chosen as the next pixel in the path, one of the two pixels that connect this neighbour with the current pixel has to be marked too in order to preserve 8-connectivity. We choose the one with the maximum gradient. Also, if two or three pixels are maximized the gradient, only one of them is choose to continue the path in order to avoid useless thickening.

Connectivity is achieved in the same raster scan as the intrinsic skeletal pixels extraction. If such a pixel is detected, we try to grow a path from it and, when completed, we continue the raster-scan. By using this technique, checking marked pixels can occur, since paths are grown in directions not yet inspected by the raster-scan too. In the case of CMD extraction (section 3), the pixel already marked are skipped by the test, whereas in the ridge point detection case (section 4 ) the pixels must be inspected anyway in order to determine whether one of its neighbours is a ridge point.

After connectivity is achieved, the skeleton still doesn't preserve the topology of the pattern, since it contains spurious holes. To guarantee correct results, a hole filling step is applied. At this point of the algorithm, the set of skeletal pixels allows a perfect reconstruction of the original pattern. But the skeleton is not unit-wide. Hence, it must be thinned. Thinning is accomplished in two steps by directly accessing the pixels through the list, which considerably reduces the time required for this operation [3].

\subsection{Pruning}

The skeleton obtained after thinning generally has many branches. Only some of them are significant for the description of the pattern, the others existing due only to the border roughness. Hence, a pruning step must be applied in order to get rid of the useless branches. Moreover, pruning is important because it considerably reduces the sensitivity of the skeleton with respect to rotation and scaling of the pattern.

Pruning is started from end points, i.e. pixels having only one neighbour. For each pixel $p$ in the branch that ends by $q$ we compute the quantity:

$$
r(p)=\frac{1}{Q}[q-p+d c(p, q)]
$$


where: $Q=A$ on square grid, and $Q=E$ on rectangular grid, and $d c(p, q)$ represents the chamfer distance between pixels $p$ and $q$, which represents the loss of information we get if we prune the branch from $q$ to $p$. If this quantity is smaller than a given threshold Th, we decide to unmark pixel $p$ and inspect the pixel next to p, and so on. Pruning is stopped for each branch when either $\mathbf{r}(\mathbf{p})$ becomes greater than the threshold, or the other end of the branch is reached and continuing to prune would disconnect the skeleton.

As for the chamfer distance dc $(p, q)$, it must be adapted to the rectangular grid. Let the coordinates of pixel $p$ be $i_{p}$ and $j_{p}$ and the coordinates of pixel $q i_{q}$ and $j_{q}$. We introduce the quantities $\mathrm{K}$ and $\mathrm{G}$ such that:

$$
\mathbf{G}=\min \left(\left|\mathrm{i}_{\mathrm{p}}-\mathrm{i}_{\mathrm{q}}\right|,\left|\mathrm{j}_{\mathrm{p}}-\mathrm{j}_{\mathrm{q}}\right|\right) \quad \mathbf{K}=\max \left(\left|\mathrm{i}_{\mathrm{p}}-\mathrm{i}_{\mathrm{q}} l,\right| \mathrm{j}_{\mathrm{p}}-\mathrm{j}_{\mathrm{q}} \mid\right)-\mathbf{G}
$$

$\mathbf{G}$ represent the number of diagonal displacements, and $\mathbf{K}$ the number of horizontal or vertical displacements between $\mathrm{p}$ and $\mathrm{q}$. With these notations, the chamfer distance adapted to the rectangular grid between pixels $\mathrm{p}$ and $\mathrm{q}$ can be written:

$$
\begin{array}{ll}
\text { if }\left|i_{p}-i_{q}\right|>\left|j_{p}-j_{q}\right| & d c=A x K+C x G+(B-C-A) x \min (K, G) \\
\text { if }\left|j_{p}-j_{q}\right|>\left|i_{p}-i_{q}\right| & d c=E x K+C x G+(D-C-E) x \min (K, G)
\end{array}
$$

Pruning must not be restricted only to the peripheral branches. Each time a connection point is reached, i.e. an intersection between two or more branches, the value of the end point $p$ as well as its coordinates are stored. Hence, when all branches intersecting in this point are pruned the connection point will become an end point. To avoid additional loss of information in this case, we compute the quantity $\mathbf{r}(\mathbf{q})$ with respect to all former end-points and we stop when one of these quantities reaches the threshold.

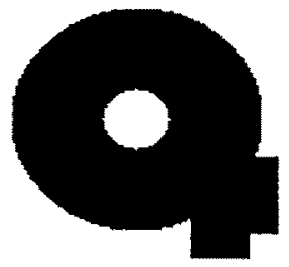

original pattern

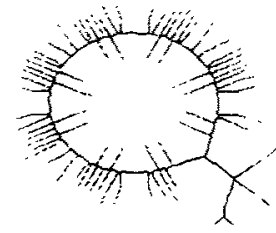

skeleton before pruning

Fig. 8. Pruning step

\subsection{Beautifying}

The aim of this step is to improve the aesthetics of the skeleton by straightening the zig-zags that appear after thinning and eliminating one-pixel branches and superfluous pixels that may remain after pruning. Details are given in [1].

\subsection{Reconstruction}

We use for the reconstruction the inverse chamfer distance transformation, which is mentioned in [10] and is achieved, as the direct transformation, in a two-pass raster scan of the image containing the skeleton. Using a $5 \times 5$ mask yields, a skeleton is more accurate and robust against rotation and scaling of the pattern.

\section{Results and comparison}

There are no great differences between the results obtained following the two meth- 
ods. They appear only for the intrinsic skeletal pixels detection, since the same steps are applied afterwards in order to determine the skeleton.

The CMD set is thicker than the ridge points one, but it contains fewer peripheral branches. As for the time required, the CMD extraction is quicker, since detecting a pixels as CMD requires fewer operations in its neighbourhood and a pixels marked in the path growing step is no longer inspected.

The dissimilarity between the original pattern and the reconstructed one can be assessed by means of the Baddeley distance [8], which is introduced in section 6.1

\subsection{Principle of the Baddeley distance}

Let $R \subset Z^{2}$ be the domain on which images are defined. Binary image $A$ (respectively B) can be regarded as a function $f_{A}: R \rightarrow\{0,1\}$ (resp. $f_{B}$ ).

$$
\begin{aligned}
& f_{A}(x, y)=1 \text { if pixel }(x, y) \text { is in the pattern } \\
& f_{A}(x, y)=0 \text { else. }
\end{aligned}
$$

Let $\mathrm{A}$ and $\mathrm{B}$ be the pattern to be compared. The Baddeley distance between $\mathrm{A}$ and $\mathrm{B}$ is defined by:

$$
D(A, B)=\left[\frac{1}{N} \sum_{p \in R}\left|d_{A}(p)-d_{B}(p)\right|^{m}\right]^{\frac{1}{m}}
$$

where $N$ is the number of pixels in $R$ and $d_{A}(p)$ (respectively $d_{B}(p)$ ) is the distance between pixel $p$ and the binary set characterizing pattern $A$ (resp.B) (Fig. 9). Index $m$ is equal to 2 in our application.

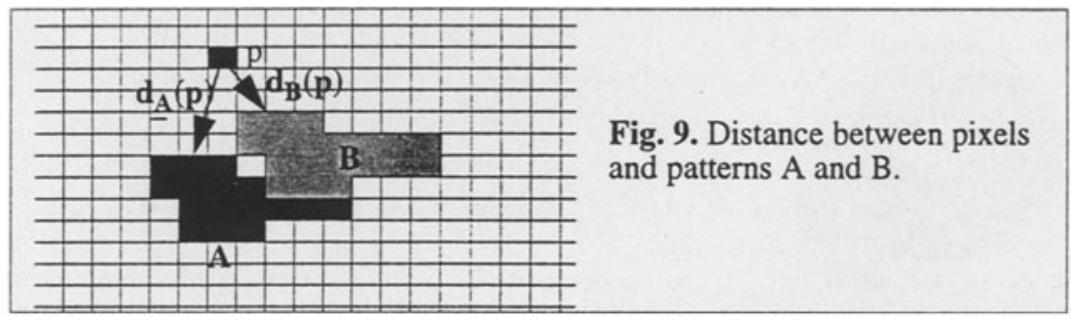

Distances $d_{A}(p)$ and $d_{B}(p)$ are computed using the chamfer distance transformation introduced in section 2 , considering the patterns $A$ (respectively $B$ ) as background in the image.

It should be noticed that, Baddeley distance $D(A, B)$ is a global criterion. It depends not only on the characteristics of the two pattern $A$ and $B$, but also on the number of pixel in domain $R$. From this point of view, the skeleton obtained using Arcelli and Sanniti di Baja's method allows a reconstruction which is more accurate, i.e. generally, the Baddeley distance of the reconstructed pattern to the original pattern is smaller for the ridge points skeleton for most of the patterns

\subsection{Experimental results}

An example is given in fig. 10. The original pattern is shown in Fig. 10a.

Fig $10 \mathrm{~b}$ and $10 \mathrm{~d}$ are obtained after applying the different steps just mentioned, and the same threshold $(\mathrm{Th}=2)$ is applied for the pruning step. The reconstructed patterns are shown in fig. $10 \mathrm{c}$ and $10 \mathrm{e} . \mathrm{D}(\mathrm{A}, \mathrm{B})$ is computed over the same domain $\mathrm{R}$. 
The best reconstruction is achieved by using the set of ridge points. This result was obtained with other patterns.

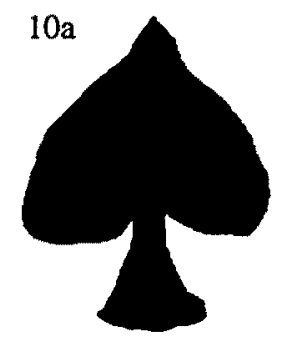

Fig. 10

$10 \mathrm{~b}$

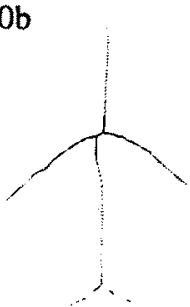

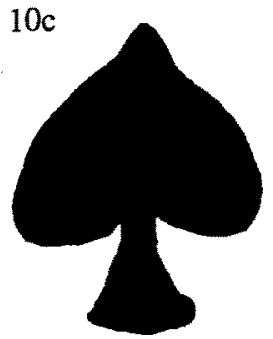

ridge point set, $T h=2, D\left(A, B_{1}\right)=2.33$

\section{Conclusion}

10a: original pattern A digitized with $\mathrm{H}=1, \mathrm{~W}=1.33$

10b: skeleton obtained starting from ridge point set

10c: $B_{1}$ reconstructed pattern $D\left(A, B_{1}\right)=2.33$

10d: skeleton obtained starting from $C M D$ set

10e: $B_{2}$ reconstructed pattern $D\left(A, B_{2}\right)=2.74$

In this paper, we have adapted the skeletonization methods described in [1] and [2] to the case of rectangular grids. By comparing them according to a quantitative criterion based on the Baddeley distance, it can be shown that the skeleton based on the ridge points extraction is better than the one based on the CMD extraction. However, the latter requires less computation time.

\section{References}

[1] E. Thiel, "Unification de la squelettisation menée en distance" 9th congrès RFIA, vol 1, pp 349-358, Paris, Janvier 1994. AFCET.

[2] C.Arcelli, G. Sanniti di Baja, "Ridge points in Euclidean distance maps". Pattem Recognition Letters, vol. 13, 1992.

[3] C.Arcelli, G. Sanniti di Baja, "Euclidean skeleton via centre-of-maximal disc extraction". Image and Vision Computing, vol. 11, 3 April 1993.

[4] D. Coquin, $\mathrm{Ph}$. Bolon, "Discrete distance operators on rectangular grids", Pattem Recognition Letters, vol. 16, pp 911-923, 1995.

[5] A. Rosenfeld, J. Pflatz, "Sequential operators in digital picture processing", J. ACM, 13, pp 471-494, 1966

[6] D. Coquin, Ph. Bolon, "Comparaison d'opérateurs locaux de distance", Proc. 3e Colloque de Geometrie Discrete: Fondements et Applications, pp 182-191, 1993

[7] G. Borgefors, "Digital transformations in digital images", Computer Vision, Graphics and Image Processing, vol. 34, pp 344-371, 1986.

[8] A.J.Baddeley, "An error metric for binary images", Robust Computer Vision, Wichmann, Karlsruhe, pp 59-78, 1992

[9] C. Arcelli, G. Sanniti, "A one-pass two operation process to detect the skeletal pixels on the 4-distance transform", IEEE Transaction Pattern Analysis, Machine Intelligence, vol.11, no. 4, pp 411-414, 1989.

[10] J.M.Chassery, A. Montanvert, "Géométrie discrète en analyse d'images". Hermes 1991. 